\title{
Interprofessional Clinical Experiences in Dental Education
}

\author{
Kristin Victoroff • Carol Savrin • Catherine Demko • \\ Jean Iannadrea $\cdot$ Sharon Freudenberger • \\ Christine Musacchio
}

Published online: 15 July 2014

(C) Springer International Publishing AG 2014

\begin{abstract}
Interest and activity in interprofessional education (IPE) has grown substantially in recent years across the health professions, driven by the belief that more effective interprofessional collaboration among providers will contribute to realization of the Triple Aim objectives articulated by the Institute for Healthcare Improvement-better individual care experiences, better population health, and reduced per capita cost of care. In dental education, new standards from the Commission on Dental Accreditation (CODA) in 2013 require that dental schools demonstrate that graduates are competent to communicate and collaborate with other health professionals, requiring dental educators to develop IPE curricula and assessment at their institutions. One of the more challenging aspects of IPE curriculum development is to provide meaningful interprofessional clinical experiences for students. The purpose of this paper is to review selected examples of interprofessional clinical experiences involving dental students and to describe an interprofessional clinical experience implemented at the authors' institution.
\end{abstract}

\author{
K. Victoroff $(\triangle) \cdot$ C. Demko $\cdot J$. Iannadrea $\cdot$ S. Freudenberger \\ Case Western Reserve University School of Dental Medicine, \\ 2124 Cornell Road, Cleveland, OH 44106-4905, USA

Keywords Interprofessional education - Clinical curriculum . Dentistry $\cdot$ Nursing $\cdot$ Dental education $\cdot$ Nursing education

\section{Introduction}

Interprofessional education (IPE) has been defined as an action that "occurs when students from two or more professions learn about, from, and with each other to enable effective collaboration and improved health outcomes" [1]. The last decade has brought significant growth in interest and activity related to IPE in health profession schools in the US and Canada. In 2010, the World Health Organization (WHO) emphasized the need for IPE and collaborative practice in order to achieve the Triple Aim objectives (Fig. 1) of improved individual experience of care, better population health, and reduced per capita cost of care articulated by the Institute for Healthcare Improvement $[1,2]$. The WHO's model (Fig. 2) postulates that IPE is critical to the development of a collaborative practice-ready healthcare workforce, and that a subsequent increase in collaborative practice will lead to improvements in the health system and improved health outcomes for the population [1].

The Institute of Medicine's 2003 report, titled Health Professions Education: A Bridge to Quality, directed attention to the need for change in the approach to educating health profession students in order to prepare a workforce with the attitudes, knowledge, and skills needed for interprofessional collaborative practice (IPCP) [3]. The report emphasized the limitations of the traditional educational model in which students are educated primarily in 'silos' with little to no interaction with their peers in other professions. The report highlighted the need for IPE in which students learn to work in interdisciplinary teams to provide patient-centered care. More recently, the Institute of Medicine's Global Forum on Innovation in Health Professional Education conducted a workshop in 2012 examining global and contemporary experiences with linkages between IPE and collaborative practice [4]. 


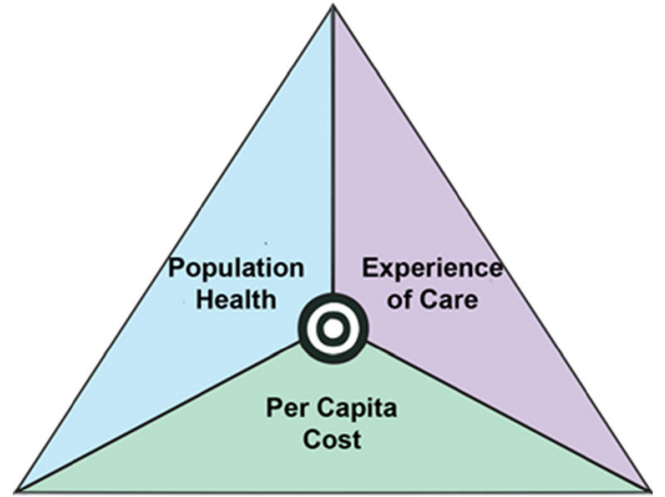

IHI Triple Aim

Fig. 1 IHI Triple Aim. IHI Institute for Healthcare Improvement

In 2011, the Interprofessional Education Collaborative (IPEC), a collaboration of the national education associations of dentistry, nursing, allopathic medicine, osteopathic medicine, pharmacy, and public health, made a stride forward in IPE by working together to articulate shared competencies applicable across the health professions [5]. These competencies address four broad areas: values and ethics for interprofessional practice; roles and responsibilities; interprofessional communication; and teams and teamwork. In late 2012, the National Center for Interprofessional Practice and Education was established at the University of Minnesota with significant funding from the Health Resources and Services Administration (HRSA) and additional support from the Josiah Macy Jr. Foundation, the Robert Wood Johnson
Foundation, the Gordon and Betty Moore Foundation, and the John A. Hartford Foundation. The center serves as a repository of resources and publications for implementing and evaluating IPE and practice across diverse settings and professions. The goal of the center is to advance IPE, with a specific focus on enhancing collaboration between health profession education and practice communities in the health system [6•].

In 2011, the American Dental Education Association (ADEA) Team Study Group on Interprofessional Education was charged with examining the status of IPE in US and Canadian dental schools. The task force published its report in 2012, emphasizing the need to produce graduates who are "fully prepared to maximize patient outcomes through interdisciplinary patient care" [7••]. On 1 July 2013, the Commission on Dental Accreditation (CODA) implemented new accreditation standards addressing IPE. The new standards require that dental schools demonstrate that graduates are competent to communicate and collaborate with other health professionals [8]. Dental education has played, and will continue to play, an important role in the development of IPE $[5,7 \bullet \bullet]$. Dental educators at all dental schools accredited by CODA must develop interprofessional curricula at their institutions in order to assist students in developing the interprofessional competencies now required by CODA. Several learning modalities can be used, including didactic instruction, simulations and clinical experiences. In order to be consistent with the competency-based educational approach required by CODA, didactic instruction alone is typically not sufficient to achieve and demonstrate competence. In addition to didactic instruction, the learner should have

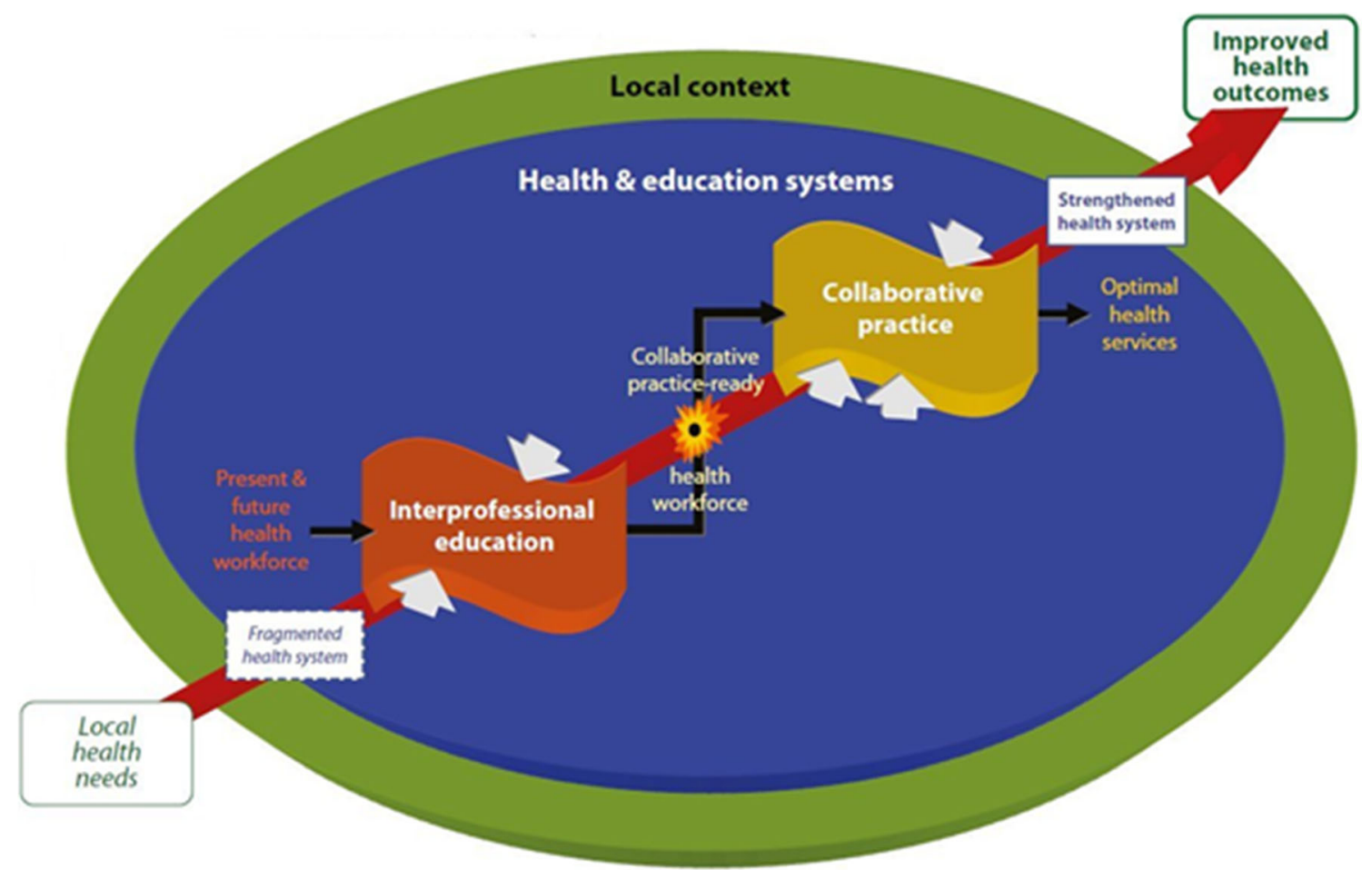

Fig. 2 Health and education systems 
opportunities to practice and to be assessed for competency in meaningful, real-world contexts [9]. In his 2012 article addressing IPE, Alfano stated that "it will take more than a didactic experience to actualize a high level of collaboration among the various health professions, and schools of health education will need to work both actively and creatively to create joint clinical training educational experiences" [10]. It can be relatively straightforward to develop an interprofessional didactic curriculum, but meaningful clinical experiences can be more challenging to develop. It is likely that students' interprofessional clinical experiences are critical for the development of interprofessional competencies and will influence the way graduates practice in the future. The purpose of this paper is to review selected examples of interprofessional clinical experiences involving dental students and to describe an interprofessional clinical experience implemented at the authors' institution.

\section{Examples of Interprofessional Clinical Experiences}

Interprofessional clinical experiences are still under development at many schools. The literature indicates that, in many cases, even schools identified as having made significant progress in IPE have only recently implemented interprofessional clinical experiences [7••]. Therefore, although information on interprofessional clinical experiences is available on schools' websites or through presentations at professional meetings, the number of published reports describing interprofessional clinical experiences involving dental students is limited at this time.

Approaches to interprofessional clinical experiences are varied. Currently reported approaches include, but are not limited to, co-location of two or more professions' clinics in close proximity to one another; integration of the professional services offered by another profession into the dental school clinic; interprofessional clinical experiences in a community setting; and integration of interprofessional student-faculty teams in the dental school clinic. Examples of each of these approaches are described below.

\section{Co-Location of Clinics in Close Proximity: New York University}

One approach involves co-location of two or more health profession clinics, a model implemented at New York University as one of a number of IPE activities at the institution [11, 12]. In 2005, the College of Dentistry and the College of Nursing at New York University developed a partnership to enhance collaboration between the two schools. In 2006, the College of Nursing opened the Nursing Faculty Practice facility within the College of Dentistry. Dental and dental hygiene students working in the dental clinic identify patients who require additional primary care services. These patients are then referred to the
Nursing Faculty Practice, which is staffed by College of Nursing faculty members who are nurse practitioners (NPs). The stated goal is for dental and dental hygiene students to act as "links to the medical network", since many patients will see a dentist and/ or dental hygienist more frequently or regularly than they will see a medical care provider. The model encourages students to recognize the need for further medical/nursing services and provide the appropriate referrals. To prepare students to address both the oral and systemic health needs of patients, and to refer appropriately, dental and NP students participate in a collaborative nursing/dental clinical rotation in the adult dental admitting center [12]. In addition, the College of Nursing has created the Oral Health Nursing Education and Practice (OHNEP) program, a national initiative that aims to enhance nurses' awareness of the importance of oral health and ability to promote oral health and refer patients who need oral health care [13].

\section{Integration of the Professional Services Offered by One Profession into the Dental School Clinic: University of Buffalo}

The University of Buffalo has implemented the second approach by integrating the professional services of social work into their dental clinic $[14,15]$. Access to and use of dental services is significantly associated with sociodemographic factors, many of which operate to decrease access and retention to dental care among traditionally underserved populations. In 1979, Levy et al. described roles for social work in university-based dental clinics, thus proposing the interprofessional collaboration between dental and social work for the benefit of patient care and students of both professions [16].

In 2001, the School of Dental Medicine and the School of Social Work at the University of Buffalo collaborated to develop and implement the Counseling, Advocacy, Referral, Education and Services (CARES) program. The core mission of the CARES program is to "improve oral health by decreasing barriers to care and improving access to dental treatment for special needs and hard-to-reach patients" [15]. Increasing patient access and retention benefits the patient, the dental student through increased hours of educational experience and types of services, and the dental school through increased revenue, principally to make the CARES program self-sustaining.

The CARES program includes two masters-level social workers providing supervision to four to seven master of social work students completing a clinical rotation. A screening protocol is utilized by dental students to identify patients who may have difficulty returning for further dental treatment. Patients receiving treatment in the CARES program generally have issues with personal finances, pain, comorbid health problems, transportation, and mental health. To facilitate knowledge transfer between the professional students, social work faculty members contribute to curriculum content 
on social and behavioral sciences for dental students, while field experience in the dental clinic is responsible for social work students' education about oral health.

The CARES program provides an opportunity for an IPE experience that is implemented in a clinical setting, enhancing students' knowledge and understanding of the benefits of a cross-disciplinary approach to improving patient care. The dental-social work model addresses both health and social barriers to increase access to dental services. While patients do not meet simultaneously with dental and social work students, the coordination of services and care supports a patient-centered model of service delivery in an educational setting which potentially can be translated to collaborative practice models. In 2009, the authors reported that $45 \%$ of CARES participants indicated, through self-report, that they would not have completed dental care without the CARES intervention [15].

\section{Interprofessional Clinical Experiences in a Community Setting: Dalhousie University}

A third approach involves interprofessional clinical experiences in a community setting. One example of this is the 'Seamless Care' model implemented by the Faculties of Medicine, Health Professions, and Dentistry at Dalhousie University. This model focused on improving students' collaborative skills through patient-centered, experiential learning [17]. Teams of students, one each from dentistry, dental hygiene, medicine, nursing, and pharmacy, collaboratively assisted patients to transition from the acute care setting in the hospital to the community. The student teams achieved their goals through an orientation workshop, meetings and educational sessions, and an 8-week clinical placement in which they participated in direct patient care. During the clinical placement, the student teams met with the patients in the acute care setting and in the patients' homes. The patientteam interactions consisted of interviewing the patients to assess their needs and preferences, and developing and implementing a plan of care that was in alignment with assessment findings and the patients' goals. Faculty from the university and professional team members from the clinic sites served as preceptors who guided and mentored the teams throughout this process. The preceptors ensured that the patients were comfortable with the student teams, interceded as needed, answered patient questions and concerns, and integrated the students' plan of care with that of the patients' primary care providers. This application allowed the students to develop interprofessional skills by gaining knowledge and experience in a clinical setting, engaging them in patientcentered care and problem solving, and by providing a professional community environment whose members modeled collaborative practice. The Seamless Care project was implemented with grant support from Health Canada.
The Seamless Care model was predicated on the goal of enhancing both learners' outcomes and the patient care experience. The model was guided by a conceptual framework grounded in principles from learning theories that give structure and organization to both the development and evaluation of the project. For example, social learning theory (SLT) [18] underlies the emphasis on learning through observation and the importance of role modeling collaborative practice by preceptors in the Seamless Care project. SLT also emphasizes the enhancement of self-efficacy through repeated practice of the team-based care tasks.

\section{Integration of Interprofessional Student-Faculty Teams in the Dental Clinic: Case Western Reserve University}

Finally, a fourth approach involves integration of interprofessional student-faculty teams in the dental school clinic. The School of Dental Medicine and the Francis Payne Bolton School of Nursing at Case Western Reserve University, the authors' institution, have implemented a program using this approach. The Collaborative Home for Oral Health, Medical Review and Health Promotion (CHOMP) program is a clinical demonstration project, implemented in early 2013, that promotes safe, efficient, and effective care, and provides health promotion, education, and prevention by integrating interprofessional student-faculty teams in the dental school clinic. The program is supported by a grant from the HRSA.

In the CHOMP model, an interprofessional team consists of an NP student, NP faculty member, dental student, and dental faculty member. The interprofessional teams work together in the dental school's Oral Medicine and Admitting Clinic, where adult patients are seen for the initial visit, and in the Pediatric Dentistry Clinic. The NP student and the dental student work together chairside to obtain a health history. The NP student can perform a limited physical examination on the patient, perform simple point-of-service tests such as glucose and hemoglobin $\mathrm{A} 1 \mathrm{C}$, provide health promotion and preventative information, provide education related to chronic illness, and administer immunizations, if agreeable to the patient. The dental and NP students huddle with the dental and NP faculty members to determine appropriate next steps for the patient and referrals as needed.

We believe that one advantage of the CHOMP model is that learners are working together chairside. Working together, with supervision from faculty members from both professions, promotes shared decision making, makes use of the opportunity for both providers to meet with patients when they are in attendance at one location, and has the potential to increase access to primary care services for the underserved population served by the dental school clinic. The intent is that NP and dental students will have the opportunity to practice skills for collaboration, such as mutual respect, communication, and 
teamwork, while also gaining knowledge related to the other's discipline. This experience provides an opportunity to learn from each other, to incorporate some of the other profession's perspective on the patient into one's own approach, and to appreciate the services the other profession provides, which may result in more frequent and informed referrals between the professions in the future. Data on billable services provided by NPs are being collected to determine the financial sustainability of the program beyond the grant funding period.

\section{Discussion}

The Triple Aim objectives of improved individual care experiences, better population health, and reduced per capita cost of care are critically important [2]. An increase in IPCP can contribute to the achievement of these objectives. IPE must prepare health profession students with the competencies necessary to effectively engage in IPCP. Development of meaningful clinical experiences that contribute to students' attainment of interprofessional competencies remains an ongoing process in dental education and across the health professions $\left[7 \bullet^{\bullet}\right.$ ]. A number of approaches have been implemented, some of which are described above. The authors are aware of institutions in which innovative interprofessional clinical experiences involving dental students are being implemented, but for which published reports are not yet available (for example, IPE at the University of Missouri, Kansas City: http://info.umkc.edu/umatters/2013/07/08/ school-of-nursing-and-health-studies-faculty-present-atinternational-interprofessional-education-conference/).

As dental educators and other health profession educators work to develop interprofessional clinical experiences for students at their particular institutions, consideration should be given to several issues. First, are there particular patient care problems or issues in the school's dental clinic, the local healthcare system, or the community for which an interprofessional approach is likely to result in progress toward the triple aim of better individual care experience, improved population health, and/or more cost-effective care? Where there is a clear problem or need to be addressed, there is likely to be greater opportunity to engage partners and to attract resources to help implement programs. If the patient is at the center of IPE, then interprofessional clinical experiences should result in improvement in the care process and/or health outcomes for the patient. For example, this was the case for the University of Buffalo's CARES model, which arose because of a particular problem (access) experienced by patients. An interprofessional approach resulted in improved access for patients $[14,15]$. Second, can the interprofessional clinical experience be sustained over time? Funding, faculty interest and availability, institutional support, and initial enthusiasm can either enhance or hamper initial efforts and then influence the continuation of the interprofessional activity. New programs must carefully consider how the clinical adjustment, if any, can be sustained over time with the same or possibly fewer resources in the future. Several of the examples described here have been sustained over a number of years, but for newer programs this is a critical question. Third, how will dental educators know that the interprofessional clinical experiences are effective in achieving the goals of IPE? Both student learning and the impact of interprofessional collaboration on patient care will need to be rigorously assessed if this question is to be answered. Does the clinical experience improve students' ability to work collaboratively and does the interprofessional collaboration improve the individual care experience, population health outcomes and/or cost effectiveness? In the CHOMP model at Case Western Reserve University, an approach to evaluation of stakeholders (faculty, students, and patients) was designed prior to the project initiation, in an effort to capture how the process occurred and any impact on the participants. Finally, the model of the future for IPCP involving dentists is not yet clear. This uncertainty presents a challenge for educators, as the extent to which educational experiences will translate to clinical practice is unclear. New models for IPCP will likely emerge over the next several years, and this is an area that the National Center for Interprofessional Practice and Education is focusing on, in order to create a bridge between education and practice.

\section{Conclusions}

IPE experiences, particularly interprofessional clinical experiences, have the potential to equip health profession students with the competencies needed for effective IPCP. Preparation of health profession students for IPCP should ultimately result in improvements in the health system and the health of the population. A number of approaches to interprofessional clinical experiences involving dental students have been implemented. Given that this is an emerging topic in dental education, it will be important for additional dental schools to share reports of their programs as they evolve.

Acknowledgments The CHOMP program at Case Western Reserve University is supported by the HRSA (grant number UD7HP25062).

\section{Compliance with Ethics Guidelines}

Conflict of Interest Dr. Kristin Victoroff, Dr. Catherine Demko, Dr. Jean Iannadrea, Dr. Sharon Freudenberger, and Mrs. Christine Musacchio each declare no potential conflicts of interest.

Dr. Carol Savrin is a consultant for various Doctor of Nursing Practice (DNP) programs. 
Human and Animal Rights and Informed Consent The CHOMP project was approved by the Institutional Review Board of Case Western Reserve University.

\section{References}

Papers of particular interest, published recently, have been highlighted as:

- Of importance

-. Of major importance

1. World Health Organization. Framework for action on interprofessional education and collaborative practice. 2010. Available from: http://whqlibdoc.who.int/hq/2010/WHO HRH HPN 10.3 eng.pdf

2. Institute for Healthcare Improvement. The IHI Triple Aim. Available from http://www.ihi.org/Engage/Initiatives/TripleAim/ Pages/default.aspx.

3. National Research Council. Health professions education: a bridge to quality. Washington, DC: The National Academies Press; 2003.

4. Interprofessional Education for Collaboration. Learning how to improve health from interprofessional models across the continuum of education to practice. Workshop summary. Washington, DC: The National Academies Press; 2013. Available from: http://www.nap. edu/openbook.php?record id=13486.

5. Interprofessional Education Collaborative Expert Panel. Core competencies for interprofessional collaborative practice: report of an expert panel. Washington, DC: Interprofessional Education Collaborative; 2011.

6. National Center for Interprofessional Practice and Education. 2012. Available from: http://www.ahceducation.umn.edu/prod/groups/ ahc/@pub/@ahc/@educ/documents/asset/ahc_asset_423392.pdf. The National Center for Interprofessional Practice and Education serves as a repository of resources and publications for implementing and evaluating interprofessional collaboration and practice.

7.• Formicola AJ, Andrieu SC, Buchanan JA, Childs GS, Gibbs M, Inglehart MR, et al. Interprofessional education in U.S. and
Canadian dental schools: an ADEA Team Study Group report. J Dent Educ. 2012;76(9):1250-68. This article will be of particular interest to dental educators. It provides a thorough overview of the rationale for dentistry's involvement in interprofessional education efforts.

8. Commission on Dental Accreditation, American Dental Association. Accreditation standards for dental education programs. 2013. Available from: http://www.ada.org/sections/ educationAndCareers/pdfs/predoc_2013.pdf.

9. Albino JE, Young SK, Neumann LM, Kramer GA, Andrieu SC, Henson L, et al. Assessing dental students' competence: best practice recommendations in the performance assessment literature and investigation of current practices in predoctoral dental education. J Dent Educ. 2008;72(12): 1405-35.

10. Alfano MC. Connecting dental education to other health professions. J Dent Educ. 2012;76(1):46-50.

11. Westphal CM, Furnari W, Haber J. College of Dentistry/College of Nursing partnership. Chicago: The American Dental Hygienists' Association; Access Jul 2009:18-21.

12. Haber J, Spielman AI, Wolff M, Shelley D. Interprofessional education between dentistry and nursing: the NYU experience. Calif Dent Assoc J. 2014;42(1):44-51.

13. Dolce MC, Haber J, Shelly D. Oral Health Nursing Education and Practice Program. Nurs Res Pract. 2012;2012:149673.

14. Zittel-Palamara K, Fabiano JA, Davis EL, Waldrop DP, Wysocki JA, Goldberg LJ. Improving patient retention and access to oral health care: the CARES program. J Dent Educ. 2005;69(8):912-8

15. Doris JM, Davis E, Du Pont C, Holdaway B. Social work in dentistry: the CARES model for improving patient retention and access to care. Dent Clin N Am. 2009;53(3):549-59.

16. Levy RL, Lambert R, Davis G. Social work and dentistry in clinical, training and research collaboration. Soc Work Health Care. 1979;5: 177-85.

17. Mann KV, McFetridge-Durdle J, Martin-Misener R, Clovis J, Rowe $\mathrm{R}$, Beanlands $\mathrm{H}$, et al. Interprofessional education for students in the health professions: the "Seamless Care" model. J Interprof Care. 2009;23(3):224-33.

18. Bandura A. Social foundations of thought and action. A social cognitive theory. Prentice Hall: Englewood Cliffs; 1986. 